Modèles linguistiques

ling uistiques

$3 \mid 2010$

Jean-Claude Chevalier. Chroniques de linguistique dans La Quinzaine Littéraire (1975-2010)

\title{
Un destin étrange
}

Jean-Claude Chevalier

\section{OpenEdition}

Journals

Édition électronique

URL : https://journals.openedition.org/ml/443

DOI : $10.4000 / \mathrm{ml} .443$

ISSN : 2274-0511

Éditeur

Association Modèles linguistiques

Édition imprimée

Date de publication : 1 décembre 2010

Pagination : 103-106

Référence électronique

Jean-Claude Chevalier, "Un destin étrange », Modèles linguistiques [En ligne], 3 | 2010, mis en ligne le

22 octobre 2013, consulté le 01 juillet 2021. URL : http://journals.openedition.org/ml/443 ; DOI :

https://doi.org/10.4000/ml.443

Ce document a été généré automatiquement le 1 juillet 2021

(c) Modèles Linguistiques 


\title{
Un destin étrange
}

\author{
Jean-Claude Chevalier
}

N 825 du 16 février 2002

Ferdinand de SAUSSURE

Écrits de linguistique générale, édités par Simon Bouquet et Rudolf Engler Gallimard, 336 p.

\begin{abstract}
Le destin de Saussure est étrange. Dès les années 1880, quand il est arrivé à Paris, jeune docteur de Leipzig, il était tenu pour un très grand comparatiste par le petit clan des linguistes, ses confrères, qui lui confièrent une Conférence aux Hautes Etudes; dans la suite, son disciple, Antoine Meillet, se vouait à la glorification du maître, retourné à Genève. Dans la première aprèsguerre, mais surtout dans la seconde, Saussure est devenu universellement célèbre, sous les espèces paradoxales d'un livre qui n'était pas de lui, mais de deux disciples, professeurs à l'Université de Genève,

Charles Bally et Albert Sechehaye.
\end{abstract}

1 Le maître étant mort en 1913 sans laisser d'œuvre d'ensemble, ils prétendaient ressusciter les grands traits de la doctrine professée pendant trois ans à Genève dans des cours de linguistique générale auxquels ils n'avaient pas eux-mêmes assisté ; ils disaient appuyer cette synthèse sur des notes d'étudiants, sur les conversations qu'ils avaient eues avec Saussure. Cette bizarre production devait désormais, sous le nom de Cours de Linguistique générale, véhiculer le message "saussurien " pour le présenter en modèle à toutes les sciences de l'homme. Ce CLG s'inscrivait dans une ambition du temps qui faisait produire des synthèses détaillées par les chefs d'école: Vendryes, Sapir, Jespersen et peu après, Bloomfield. À l'inverse, Saussure appartenait à cette génération plus ancienne de comparatistes qui réfléchissaient sur les lois générales du 
langage, mais se refusaient à produire un système général. Y serait-il venu ? C'est peu vraisemblable ; au reste, la mort l'avait frappé prématurément, en 1913.

Discordance sensible. Dès la publication du CLG, Antoine Meillet, dans son compte rendu du Bulletin de la Société de Linguistique, avait signalé qu'il ne reconnaissait pas le ton - ni l'esprit - du Saussure qu'il avait connu, son brio, ses hésitations, son sens de la métaphore, ses doutes. Un peu plus tard Nicolas Troubetzkoy, nouvelle vedette à Prague et Vienne, exprimait ses réserves. Le livre était trop bien organisé, trop fermé, trop péremptoire (et c'est sans doute à quoi il devrait son succès). Mais il fallut attendre 1957 et Robert Godel pour qu'on ait la curiosité de remonter aux sources et de dessiner une figure nouvelle de Saussure. L'économie du CLG n'était pas en cause, mais la démarche intellectuelle d'un comparatiste qui proclamait volontiers qu'il n'était pas possible d'écrire à la suite deux lignes sensées de linguistique générale; et qui donc multipliait les notes et esquisses. On recensa une masse de papiers dispersés qui nourrirent alors des éditions critiques du CLG, l'admirable travail de Tullio di Mauro (1972) et la somme érudite de Rudolf Engler; on exhuma des comptes rendus avortés (comme celui que Saussure voulait consacrer aux Programme et Méthodes de la linguistique théorique d'Albert Sechehaye en 1908), des articles d'hommages jamais envoyés (tel celui qu'il destinait à glorifier Whitney, son modèle américain), des extraits d'« une théorie complète de la syllabe » qui n'était pas complète (244 sv.), des notes critiques ; telle cette dénonciation de la pauvreté de la métaphore du jeu d'échecs, célèbre en grammaire historique :

Une langue n'est comparable qu'à la complète idée de la partie d'échecs, comportant à la fois les positions et les coups, à la fois des changements et des états dans la succession... (207)

3 Textes non finis, troués, qui laissaient place à l'imaginaire et à d'ambitieuses constructions seulement suggérées, tentations et tentatives.

D'autres chercheurs réveillèrent d'autres sources, plus complètes et plus proches de la parole saussurienne, les notes prises au cours : les trois cours de Genève, professés à partir de 1906, ont été reconstitués tout récemment par Eisuke Komatsu et Georges Wolf. Un chercheur italien, Maria-Pia Marchese, a même pu reconstituer une Phonétique d'après un manuscrit dit de Harvard, déjà commenté par R. Jakobson et $\mathrm{H}$. Parret.

5 Ainsi, par touches, est apparu un Saussure assez différent de celui du CLG, beaucoup moins didactique, plus fulgurant, moins idéaliste, plus suggestif, plongeant au plus profond du langage, plus "philosophe", comme il disait, animé par une sévère exigence intellectuelle :

(Il faut) s'élever au degré d'abstraction qui est nécessaire pour dominer d'une part ce qu'on fait, d'autre part en quoi ce qu'on fait a une légitimité et une raison d'être dans l'ensemble des sciences. (205)

6 C'est ce personnage qu'a voulu faire apparaître Simon Bouquet, auteur d'une superbe Introduction à la lecture de Saussure, 1997, en éditant, avec son maître R. Engler, ces Écrits de linguistique générale. $\mathrm{SB}$ et $\mathrm{RE}$ ont conjoint des fragments déjà connus et des textes nouveaux. La pièce de résistance, c'est un manuscrit inédit d'environ 70 pages découvert il y a peu lors de travaux dans l'Orangerie de la demeure patricienne des Saussure à Genève ; il portait en mention « De l'essence double du langage ». C'est peutêtre le livre de méditations dont parlait Saussure en 1911 à un de ses étudiants, assemblage composite, inachevé, dominé par quelques idées fortes. Ainsi, que toute 
analyse linguistique repose sur des oppositions, qui dégagent des valeurs. À l'opposé du matériel des sciences, jamais une entité n'est simple puisqu'il faut tenir compte à la fois du signe et de la signification :

Il est faux (et impraticable) d'opposer la forme et le sens. Ce qui est juste, en revanche, c'est d'opposer la figure vocale d'une part et la forme-sens d'autre part.

Système en perpétuel rééquilibrage, fondé sur des différences qui jouent comme des négativités. On ne sait même pas comment appréhender le changement :

Parler de changement serait supposer qu'il y a des états stables dans la langue, ce qui n'existe pas. (60)

Notes laconiques, souvent surprenantes : le linguiste est pris entre la crainte de ne dire que des banalités ou d'endosser le ridicule d'être paradoxal. (61)

9 Ce qui est saisissant, c'est la rigueur de la réflexion sur le fonctionnement du langage qui rêve parfois d'un recours à la logique. La phonétique - et constamment la phonétique du sanscrit - est le champ d'exercice favori de son épistémologie, alors que dans le CLG, elle sera intégrée dans le traitement de l'ensemble. On retrouve ce même paradoxe chez Chomsky dont l'étude des Syntactic Structures et autres semblables a répandu la gloire et qui, lui, fut longtemps obsédé par les apories exposées dans Sound Pattern. Même souci de la rigueur dans la création de caravanes pittoresques de termes nouveaux : le sème, le parasème et l'aposème, l'inertôme, le sôme, le contre-sôme, le parasôme et l'anti-sôme et le sôma, etc. Si nombre de ces néologismes ont disparu aussitôt que créés, plusieurs ont conduit à clarifier durablement la pensée linguistique, comme sémiologie qu'Ad. Naville, à la grande fierté de Saussure, avait retenu dans sa Classification des Sciences. Et faut-il redire que signifiant et signifié n'apparaissent que dans le Troisième Cours. À quelques mois près, on perdait un binôme qui a tant fait pour la diffusion de la linguistique.

Le texte de l'«Essence double » est flanqué par les éditeurs de brouillons, d'exposés, certains inédits, d'autres, plus nombreux, déjà reproduits par des archivistes antérieurs et par R. Engler, en particulier. L'ensemble donne parfois l'impression d'un ressassement, comme si Saussure éprouvait une exaltation à approcher certains mystères de la langue. Même si parfois un passage fulgurant illumine des retournements angoissés. Tel : "Le signe est un fait de conscience pur " (19) ou «Le rapport entre le trait graphique et le son parlé est le même qu'entre le son parlé et l'idée » (49). Textes étranges si éloignés du maîtrisé CLG. Le lecteur est au Centre du laboratoire central du langage critique, proche de la pensée pure, de la poétique du savoir. Ivresse d'autant mieux garantie que les éditeurs, « Préface » faite, ont renoncé à toute annotation. Le lecteur s'abandonne avec ravissement au flux saussurien comme d'autres fois il s'est abandonné au flux wittgensteinien, il sent le flux du génie. 